\title{
BMJ Open Qualitative study using interviews and focus groups to explore the current and potential for antimicrobial stewardship in community pharmacy informed by the Theoretical Domains Framework
}

To cite: Jones LF, Owens R, Sallis A, et al. Qualitative study using interviews and focus groups to explore the current and potential for antimicrobial stewardship in community pharmacy informed by the Theoretical Domains Framework. BMJ Open 2018;8:e25101. doi:10.1136/ bmjopen-2018-025101

- Prepublication history and additional material for this paper are available online. To view these files, please visit the journal online (http://dx.doi org/10.1136/bmjopen-2018025101).

Received 3 July 2018 Revised 18 October 2018 Accepted 23 November 2018

Check for updates

(C) Author(s) (or their employer(s)) 2018. Re-use permitted under CC BY-NC. No commercial re-use. See rights and permissions. Published by BMJ.

For numbered affiliations see end of article.

Correspondence to Dr Cliodna A M McNulty; cliodna.mcnulty@phe.gov.uk

\section{ABSTRACT}

Objectives Community pharmacists and their staff have the potential to contribute to antimicrobial stewardship (AMS). However, their barriers and opportunities are not well understood. The aim was to investigate the experiences and perceptions of community pharmacists and their teams around AMS to inform intervention development.

Design Interviews and focus groups were used to explore the views of pharmacists, pharmacy staff, general practitioners (GPs), members of pharmacy organisations and commissioners. The questioning schedule was developed using the Theoretical Domains Framework which helped inform recommendations to facilitate AMS in community pharmacy.

Results $8 \mathrm{GPs}, 28$ pharmacists, 13 pharmacy staff, 6 representatives from pharmacy organisations in England and Wales, and 2 local stakeholders participated. Knowledge and skills both facilitated or hindered provision of self-care and compliance advice by different grades of pharmacy staff. Some staff were not aware of the impact of giving self-care and compliance advice to help control antimicrobial resistance (AMR). The pharmacy environment created barriers to AMS; this included lack of time of well-qualified staff leading to misinformation from underskilled staff to patients about the need for antibiotics or the need to visit the GP, this was exacerbated by lack of space. AMS activities were limited by absent diagnoses on antibiotic prescriptions. Several pharmacy staff felt that undertaking patient examinations, questioning the rationale for antibiotic prescriptions and performing audits would allow them to provide more tailored AMS advice.

Conclusions Interventions are required to overcome a lack of qualified staff, time and space to give patients AMS advice. Staff need to understand how self-care and antibiotic compliance advice can help control AMR. A multifaceted educational intervention including information for staff with feedback about the advice given may help. Indication for a prescription would enable pharmacists to provide more targeted antibiotic advice. Commissioners should consider the pharmacists' role in examining patients, and giving advice about antibiotic prescriptions.

\section{Strengths and limitations of this study}

- This is the first study to explore pharmacists' and pharmacy staff attitudes and experiences around self-care advice for common infections, antibiotic compliance advice, antimicrobial stewardship activities and antimicrobial resistance (AMR) using the Theoretical Domains Framework (TDF).

- The use of the TDF and Behaviour Change Wheel is a novel use of behavioural theory in this context.

- Randomly selecting pharmacies from stratified lists facilitated recruitment of a wide-ranging sample with a range of experiences.

- Despite using randomised lists for recruitment, there is still a possibility that only AMR enthusiasts volunteered to take part.

- This study used focus groups and interviews including a mix of telephone and face-to-face methods. This ensured that participants could choose the method which was most convenient and appropriate for them.

\section{INTRODUCTION}

WHO has reported concerns about the threat of antibiotic resistance and stressed the need for public awareness of the responsible use of antibiotics. ${ }^{1}$ Patients have an important role to play in the control of antibiotic resistance, by reducing use of unnecessary antibiotics for common infections (such as for respiratory infections) and by adhering to their prescription instructions when they do receive antibiotics. $^{2}$ Community pharmacists can facilitate this through their contact with patients and by promoting antimicrobial stewardship (AMS) initiatives within their pharmacies. ${ }^{3}$ Community pharmacists have the opportunity to influence patient expectation by educating patients on effective self-care treatments and the negative consequences of using antibiotics, such as side effects and resistance. ${ }^{4}$ 
Within England, the Department of Health (DOH) pharmacy strategy 2005-2015 aimed to enable community pharmacists and their staff to see themselves as important contributors to improving public health. ${ }^{5}$ The DOH is currently incentivising community pharmacies (through the community pharmacy contractual framework) to meet criteria to expand and improve their range of clinical services. One of these criteria is to become a Healthy Living Pharmacy level $1,{ }^{6}$ through demonstrating that the pharmacy team is actively engaging with the local community. ${ }^{7}$ In addition, the National Health Service (NHS) in England has been expanding the services already provided by community pharmacies to include treatment for urgent minor ailments and common conditions including viral infections. ${ }^{6}$ Indeed, research has shown that through various strategies community pharmacists can enhance their role in AMS, ${ }^{8}$ by providing selfcare and antibiotic compliance advice to patients ${ }^{9}$ as well as the ability to recommend over-the-counter treatments for common infections. ${ }^{10}$ However, this incentive scheme is not available nationally in England, therefore, there is considerable variation in community pharmacy services.

Within Wales, the Choose Pharmacy scheme encourages the general public to seek advice and treatment for minor ailments from community pharmacies. The scheme also aims to fully integrate community pharmacies with general practitioner (GP) surgeries and hospitals by linking their technology, such as providing pharmacy access to GP records in order to check the accuracy of prescribed medications. ${ }^{11} 12$

The aim of this study is to identify opportunities for expanding AMS activities by exploring pharmacists' and pharmacy staff attitudes and experiences around selfcare advice for common infections, antibiotic compliance advice, AMS activities and antimicrobial resistance (AMR). The findings from this study will contribute to recommending intervention strategies for community pharmacy teams in order to enhance their role in helping to control AMR.

\section{METHOD}

This was a qualitative study using semistructured interviews and focus groups with community pharmacists, community pharmacy staff, GPs, pharmacy body representatives and commissioners.

\section{Community pharmacy selection}

UK pharmacies in Inner city Birmingham, Gloucestershire and South Wales were approached to recruit pharmacies with a mix of deprivation, ethnicity, rural and urban locations, varying customer numbers and customer demographics. Pharmacies in each area were stratified by rural/urban based on their postal code details ${ }^{13}$ if relevant, and in order to obtain pharmacies with varying staffing levels and a range of corporate resources available, by chain/small chain/independent using a Google search of the pharmacies (later confirmed in the interview/ focus group). Each stratified list was randomised using the random number generation function in Excel, and approached in random order by letter and then telephone until the required number of participants for each region and pharmacy type was reached. The target was to recruit 30 pharmacists and 8 pharmacy staff from across the regions and strata.

\section{Participant recruitment}

Pharmacies were sent invitation letters, study information sheets and a consent form inviting pharmacists and pharmacy staff to participate in interviews or focus groups for the study. If no response was received after a week, the researchers telephoned the pharmacy. The most common reason for non-participation was lack of time.

Representatives from pharmacy body organisations, GPs and Clinical Commissioning Groups (CCGs-CCGs are state funded and commission primary healthcare from general practices in England), were invited via contacts known to the researchers and by means of a Royal College of General Practitioners newsletter to also participate in interviews (see figure 1).

\section{Data collection}

The question schedule was drafted by a researcher (LFJ) and then adapted by a health psychologist (AS) using the Theoretical Domains Framework (TDF) in order to understand the influences on participant behaviour, ${ }^{14}$ and then reviewed by other researchers (CAMM, LFJ and RO) for clinical relevance. The question schedule (online supplementary appendix 1) was piloted with one pharmacist. There were no changes made following the pilot and so the results from the pilot are included in the findings. The broad topic areas discussed were interviewees' attitudes and experiences of providing self-care advice for common infections, and antibiotic compliance advice, AMS activities and AMR. One trained qualitative researcher (LFJ) from Public Health England (PHE) conducted the interviews and focus groups. All participants were assured of anonymity, confidentiality and gave written informed consent. Participants were offered a $£ 40$ gift voucher for their time.

Focus groups were conducted within pharmacies where several members of staff expressed an interest in taking part. Interviews were conducted by telephone and face to face in order to accommodate participant preference. They lasted 30-60 min, were audio recorded, transcribed verbatim and checked for accuracy.

\section{Data analysis}

Data were analysed by a PHE researcher, LFJ, using a thematic framework and using QSR NVivo V.10. Themes were refined and redundant or infrequent codes were recoded. The remaining themes were placed within the TDF framework with assistance from a health psychologist (AS), and where more than one domain was relevant the context was discussed and a consensus reached. Quotations from the transcripts are used in the results table to 


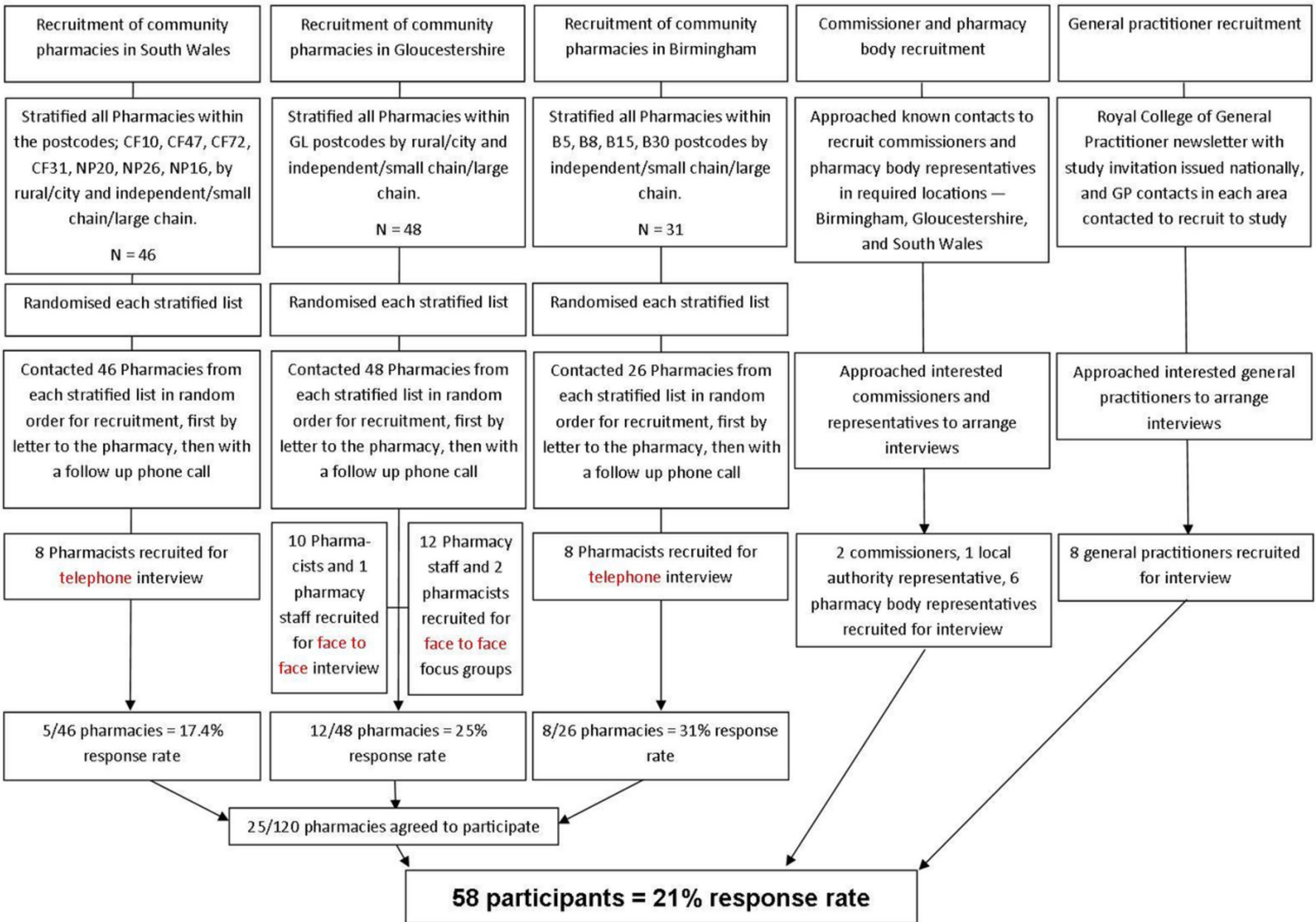

Figure 1 Recruitment flow chart. The recruitment flow chart demonstrates the method and process of recruitment for each participant group.

illustrate each domain. A researchers meeting was held to discuss the main themes from the results and to discuss themes to take forward for intervention recommendations. These were examined within the context of the Behaviour Change Wheel $(\mathrm{BCW})^{15}$ in order to identify intervention functions, policy categories and behaviour change techniques (BCTs) relevant for intervention recommendations and future developments.

Ten per cent of the transcripts were double coded by a second PHE researcher. Codes were discussed and an agreed consensus was reached.

\section{Patient involvement}

Patients were not involved in this study.

\section{RESULTS}

\section{Sample characteristics}

Twenty-six out of 31 pharmacies were contacted in Birmingham. All pharmacies in the Gloucestershire and South Wales lists were approached in order to achieve the recruitment targets.

A total of 58 people took part in either interviews or focus groups for this study. Forty-four interviews were conducted with 26 community pharmacists (10 from Gloucestershire, 8 from Birmingham and 8 from South Wales), 1 dispensing manager, 8 GPs (3 from Birmingham, 1 from Gloucestershire, 2 from Hertfordshire, 1 from London and 1 from Colchester), 2 primary care commissioners, 1 programme manager from a local authority and 6 representatives from pharmacy organisations (Royal Pharmaceutical Society, Pharmacy Voice, The Centre for Pharmacy Postgraduate Education, The Pharmaceutical Services Negotiating Committee, Association of Pharmacy Technicians UK, NHS England and the National Pharmacy Association).

Three focus groups were conducted in three pharmacies, with 2 pharmacists and 12 other pharmacy staff. Other pharmacy staff included five dispensers, one pharmacy manager, one dispensing assistant, two healthcare assistants and one shop assistant.

Of all pharmacy participants, 16 worked in an independent pharmacy (a pharmacy which is independently owned), 5 in a small chain, 18 worked for a large chain (a chain refers to more than one pharmacy which is centrally managed or owned) and 2 pharmacists were locums. Pharmacists were aged 24-63, with a mean age of 41. Eighteen were male and 10 were female. 
The analysis identified key domains from the TDF on influencing pharmacists' behaviour within community pharmacy which are relevant to our study, as outlined in table 1 .

\section{Giving self-care advice in community pharmacy}

Pharmacists reported that giving self-care advice daily for common infections is an integral part of their role, and by using the WWHAM mnemonic, ${ }^{16}$ who is the patient, what are the symptoms, how long have the symptoms been present, action taken, medication being taken, as well as their experience. Pharmacists identified some difficulty in determining the need for antibiotics in the elderly or the very young. When pharmacists are uncertain or believe they are dealing with a serious condition, they refer the patient to their GP, or if a patient evidently needs immediate care they will refer to urgent care.

A minority of GPs and one pharmacy body representative expressed concern that pharmacists are financially motivated in the advice they give and the products which they recommend. Pharmacists reported that their main motivation is the health and well-being of their patients, not financial incentives.

The stated benefits of providing self-care in community pharmacy included immediate access to a health professional, equipping patient with knowledge for future infective episodes and saving GP time. Indeed, pharmacists reported that they try and discourage patients visiting the GP, and try and promote self-care when possible. Most believe that educating patients with self-care advice can contribute to tackling AMR by preventing future antibiotic use. For some, preventing future antibiotic use was their main motivation, some, however, were not aware of this link until it was mentioned by the researcher.

The GPs in this study view pharmacists as being knowledgeable about medications and sufficiently skilled to make recommendations to patients. As such, they were confident in the ability of pharmacists to deal with minor respiratory tract infections.

\section{Barriers to giving self-care advice in community pharmacy}

Pharmacists and other pharmacy staff identified that pharmacy staff usually have more interactions with patients looking for self-care advice as pharmacists spend little of their time at the medicines counter in large pharmacies. Pharmacists expressed concern that misinformation can be given to patients if their staff are untrained or inexperienced. Lack of time was perceived as a barrier to giving effective and thorough self-care advice and can lead to advice being given quickly over the counter.

Some pharmacists felt that being unable to examine patients (eg, listen to their chest) is a barrier to giving accurate self-care advice. They believe that being able to conduct such examinations would enhance the service they could provide. One pharmacist highlighted a concern that focusing too much on providing self-care advice could potentially lead to not identifying a more serious infection. Despite pharmacists reporting that they receive informal feedback on their advice from many of their regular patients, many identified a lack of formal procedure to receive feedback on their self-care or compliance advice given. Some pharmacists believed that patients want an instant cure for their ailments and are not willing to wait for the duration of their illness to feel better.

\section{Giving antibiotic compliance advice in community pharmacy}

Pharmacists, their staff and GPs reported that a key responsibility in community pharmacies is to provide antibiotic advice and ensure patient compliance. Among pharmacists, the idea that misuse of antibiotics results in resistant infections was well understood. For some, AMR was a concern and therefore a driver for giving compliance advice. Less qualified pharmacy staff did not display an in-depth understanding of AMR but were aware that misuse of antibiotics can lead to recurrent or relapsing infections.

\section{Barriers to giving antibiotic compliance advice in community pharmacy}

Pharmacists are not provided with an indication of patients' diagnoses on prescriptions, and this was reported by some as a major barrier as it deters pharmacists from querying the accuracy of antibiotic prescriptions with prescribers. It was reported that when giving compliance advice, it would be unusual for a pharmacist to ask what condition the antibiotics had been prescribed for.

Pharmacists reported that many patients do not understand compliance advice, and therefore, believe that compliance is generally poor. Additionally, pharmacy staff noticed that some patients tend to be disinterested in the compliance advice.

\section{AMR and AMS in community pharmacy}

Pharmacists were generally unaware of any stewardship initiatives from within and outside of the community pharmacy setting. Many had never heard of the term AMS before. Despite this lack of awareness, when AMS was explained pharmacists believed they had a role in tackling AMR through educating the general public on how to self-care for common infections and the appropriate use of antibiotics.

Pharmacists in Wales had conducted self-care audits in their pharmacy which they felt had raised awareness of AMR and had contributed to improvements in their practice.

All pharmacists expressed concern about AMR and its implications. However, no one reported any intentions to adopt any AMS promotions or activities in their pharmacy in the foreseeable future.

\section{Barriers to AMS in community pharmacy}

The biggest barrier identified was that many pharmacists and all pharmacy staff were not consciously aware of the link between giving self-care advice, compliance advice and their impact on AMR. Many only became aware of the link once it was brought to their attention by the 


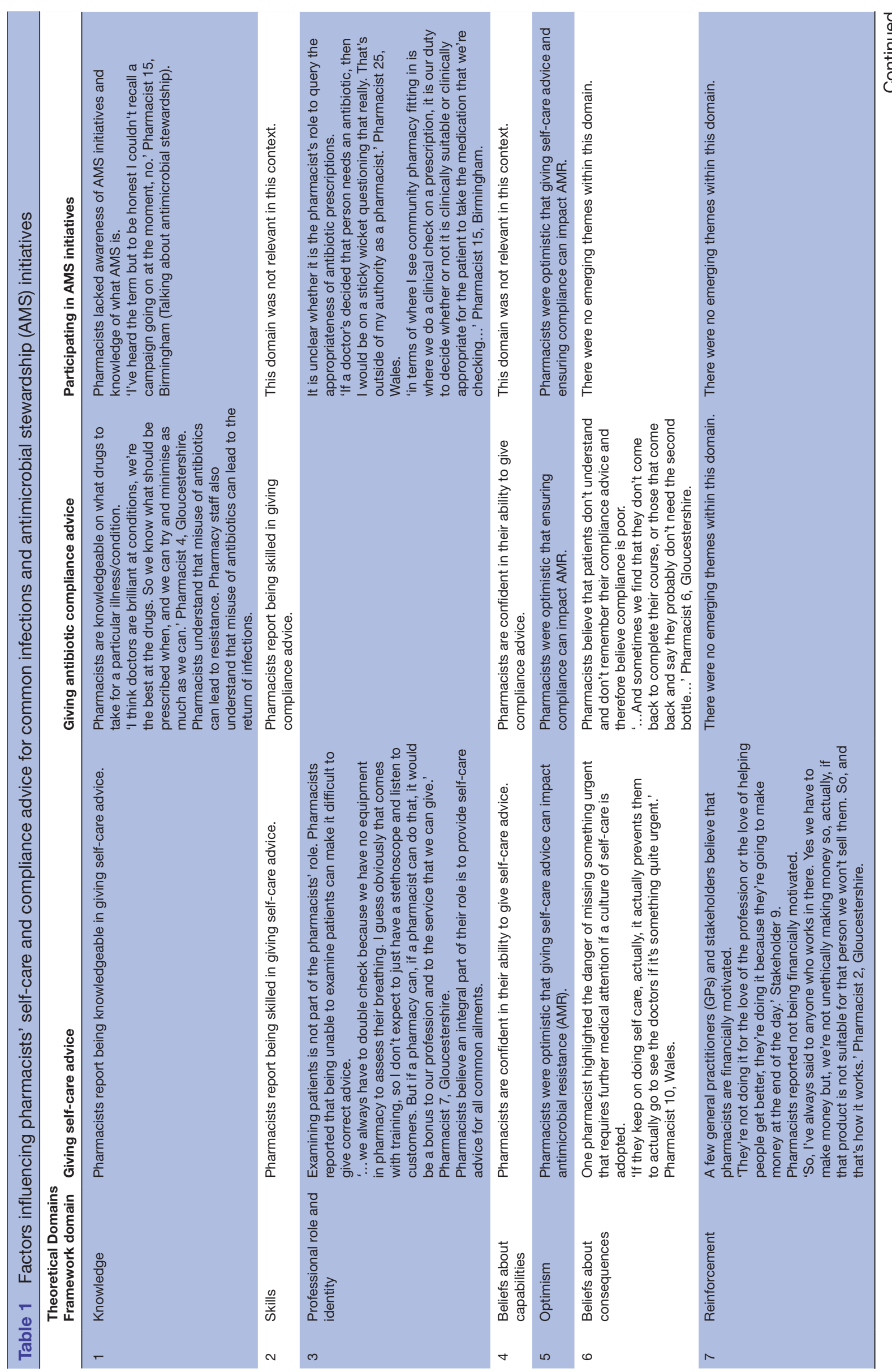




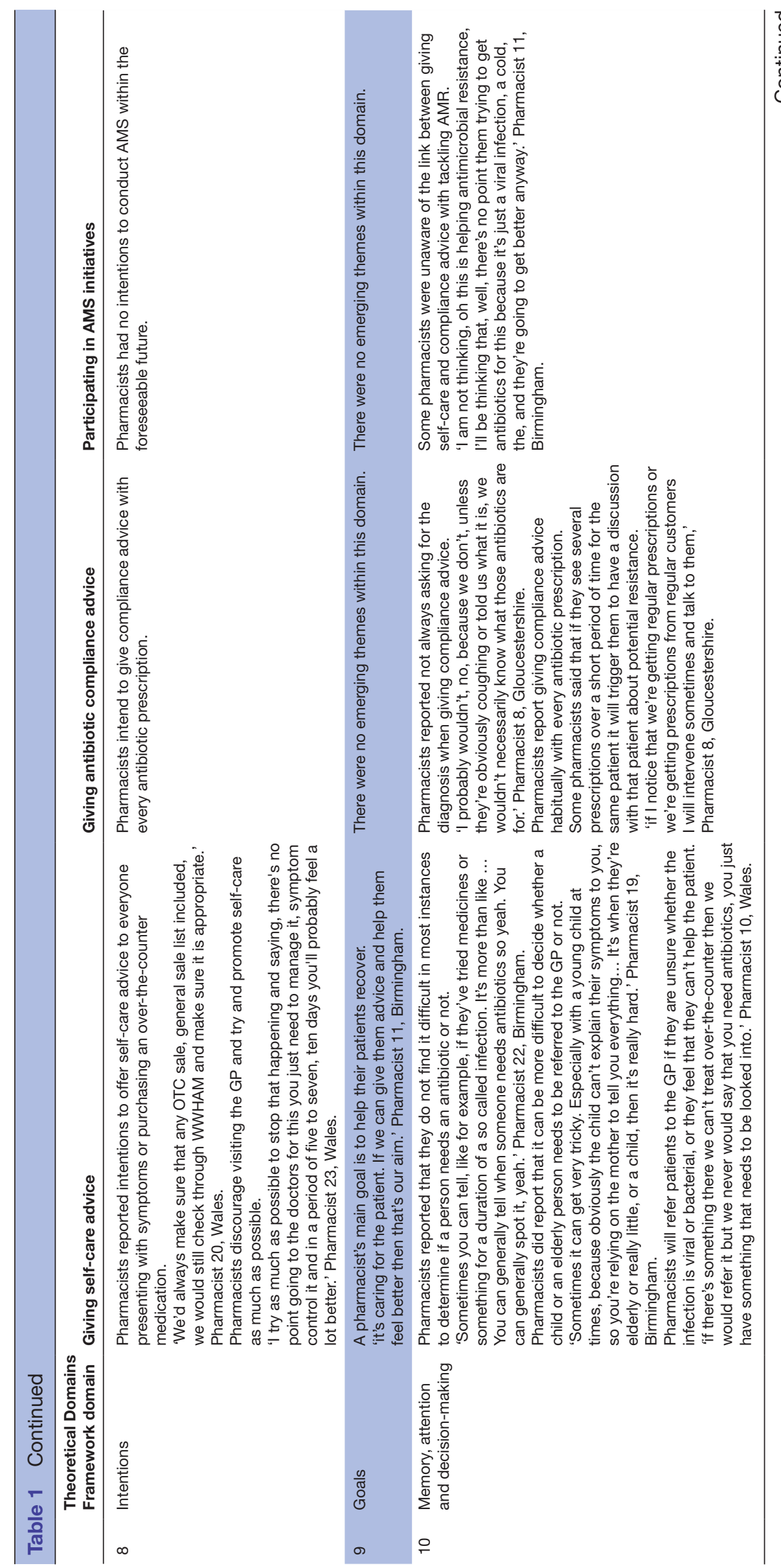




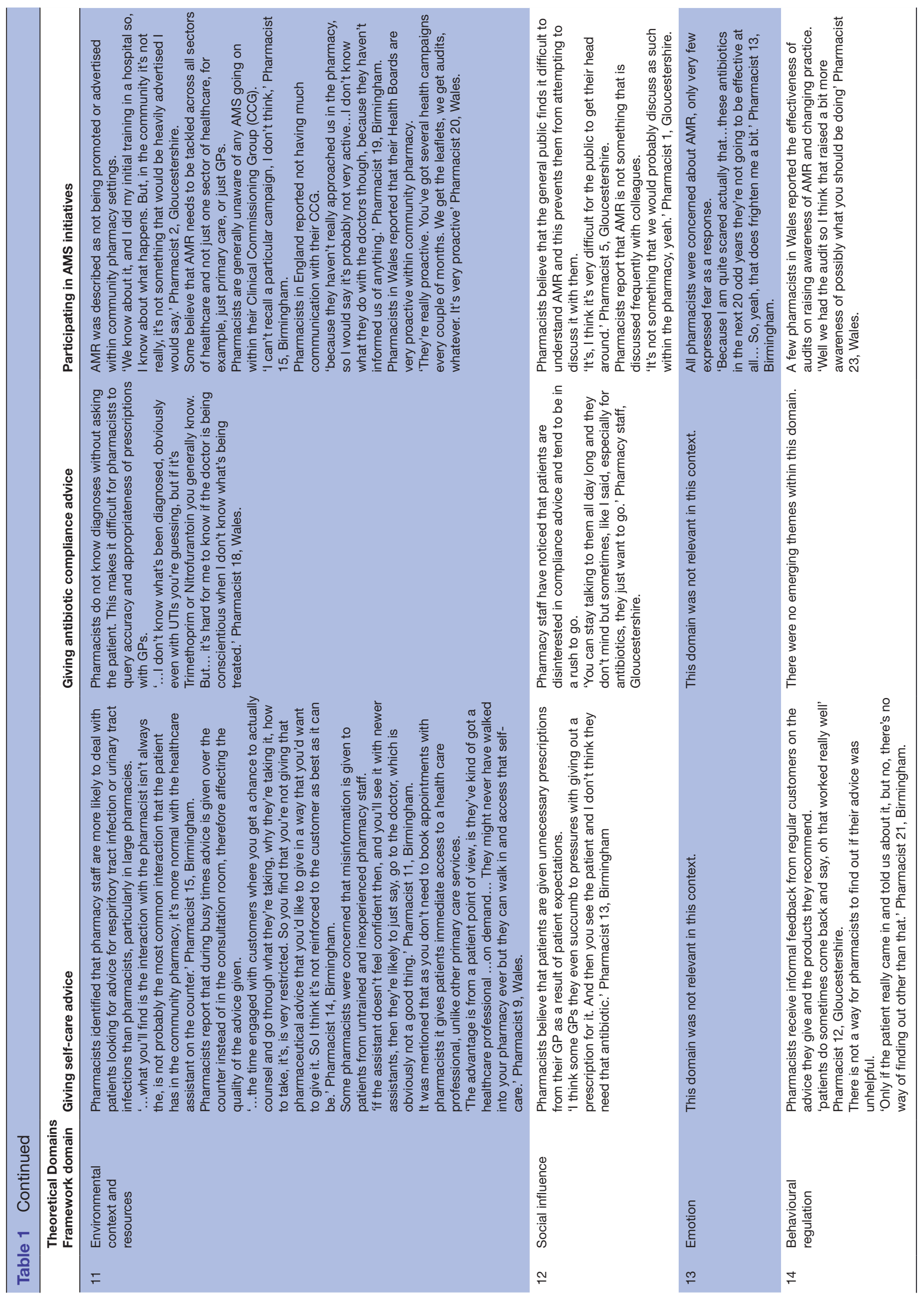


interviewing researcher. Once aware, they were optimistic that giving self-care advice and compliance advice could have a significant impact on AMR. Although, many pharmacists believed that discussing AMR with the general public would be difficult as their perception was that they would find it difficult to understand the concept.

\section{Summary}

Identification of potential interventions

Analysis using the TDF has been applied here to the BCW. ${ }^{15}$ By identifying key influential domains from the TDF, the BCW allows for the identification of intervention functions, policy categories and alongside the BCT Taxonomy v1, BCTs to inform intervention recommendations in this context. Table 2 demonstrates this process of identifying key domains through to intervention recommendations.

\section{DISCUSSION}

\section{Implications for practice}

Barriers such as underskilled staff giving misinformation to patients and time pressure both for patients and pharmacists are reported to be influential on the quality of advice given to patients. Therefore, time-saving resources need to be developed to assist pharmacists and pharmacy staff in giving self-care advice and antibiotic compliance advice to patients that can also double as educational resources for new pharmacy staff or pharmacy staff in training. Feedback from this study indicates that the TARGET Treating Your Infection leaflet for pharmacies could be an appropriate solution, as shown by a recent pilot study of the TARGET leaflet in community pharmacy which concluded that the leaflet will act as a cue to have infection-related self-care conversations with patients and facilitate a short consultation. ${ }^{17}$ Adapting the TARGET UTI leaflet or use of other pictorial leaflets may also help. ${ }^{18}{ }^{19}$ Pharmacy staff also suggested pictorial stickers or simple leaflets in order to communicate and reinforce compliance advice with patients.

Audit templates to assess self-care and antibiotic compliance advice in pharmacies should increase awareness of the link between giving self-care advice and AMR, and improve the pharmacy service. Any such audits should provide firm action plans and allow for reauditing with the aim of quality improvement. ${ }^{20}$

If the role of pharmacists is to consider the accuracy of antibiotic prescriptions or give the most appropriate selfcare advice then they need to be aware of patients' diagnoses. Without diagnosis information, pharmacists are only able to identify that the prescription is the correct course and dosage for the specified antibiotic and give appropriate compliance advice. A system should be developed to provide pharmacists with quick and easy access to prescription indications which will support pharmacists in their AMS activities, enabling them to provide tailored self-care advice alongside antibiotic prescriptions. Selfcare advice will facilitate patients self-care for any future self-limiting infections. Providing coded diagnoses, similar to that of Read coding, offers a quick and confidential way of communicating diagnosis information to pharmacists. An alternative could be to provide pharmacists with access to patient records in order to access diagnosis. Indeed the Royal Pharmaceutical Society believes that pharmacists should have full access to patient records in the interest of safe and effective patient care. ${ }^{21}$ However, in community pharmacy, time would need factoring into the dispensing process to enable community pharmacists to check clinical appropriateness within local guidelines for each antibiotic prescription.

Extending pharmacy services to include patient examination could be an optional additional training for pharmacists to enable more targeted advice. Further research would be required to evaluate the additional service to understand the added benefit versus risks to the wider health economy and patients.

\section{Implications for research}

It is recommended that future research uses behavioural theory such as the TDF and the BCW in the development of AMS interventions for community pharmacy, in order to understand the behavioural domains, intervention functions and policy categories relevant to successful implementation. It will be important to develop any interventions alongside input from target users such as pharmacists, pharmacy staff and stakeholders, including the general public for any patient facing interventions; in order to understand potential barriers and facilitators to implementation, and any recent changes to perceptions of AMR and AMS since this study. The effect of any interventions or resources needs to be evaluated in a range of pharmacy settings.

\section{Comparison with existing literature}

A recent audit of over-the-counter medication sales and self-care advice demonstrated that community pharmacies are the first port of call for patients. During the audit period, over 4 in 10 instances where an over-the-counter product was not supplied was because the community pharmacy team identified that the patient required a referral to another health service, the most common being GP referrals. ${ }^{22}$ With lack of time and staff skillset being an issue, it is recommended here that a resource is developed to support pharmacists and their staff in giving effective self-care and compliance advice.

GPs and pharmacists both reported that common infections can be dealt with in community pharmacy as pharmacists are knowledgeable and well skilled in providing effective advice. Indeed, research has shown that patients are satisfied with consultations by non-medical prescribers such as pharmacists for acute respiratory tract infections. ${ }^{23}$ Forty-four per cent of patients expected a physical examination from their non-medical prescriber and reported that it was important for reassurance. Similarly, non-medical prescribers used physical examinations to reassure patients, and as a form of evidence to justify 





their treatment decision. ${ }^{23}$ While pharmacists are not trained to listen to patient breathing it was reported by some pharmacists in this study that it would be a useful addition to community pharmacy services.

\section{Strengths and limitations}

To our knowledge, this is the first study to explore pharmacists' and pharmacy staff attitudes and experiences around self-care advice for common infections, antibiotic compliance advice, AMS activities and AMR using the TDF. The use of the TDF and BCW to guide the question schedules, interpret the findings and inform intervention recommendations is a novel use of behavioural theory in this context.

By recruiting pharmacists and pharmacy staff through randomly selecting pharmacies from lists stratified by urban/rural, independent/small chain/large chain, facilitated recruitment of a wide-ranging sample with a range of experiences. Some had hospital experience and therefore had the first-hand experience of the implications of bacterial resistance, some had only worked in small independents and therefore had close relationships with their community and others worked with large teams in high street pharmacies with fewer familiar patients. As with qualitative studies, there is always the possibility that only AMR enthusiasts volunteered to take part, however, the study team believe that randomisation of the pharmacy lists and the $£ 40$ financial incentive to participate enabled and attracted non-enthusiasts to participate. Furthermore, pharmacy staff admitted not understanding the link between self-care and compliance with AMR indicating that they were probably not enthusiasts.

This study used focus groups and interviews including a mix of telephone and face-to-face methods. This ensured that participants could choose the method which was most convenient and comfortable for them. It could be argued that a large scale survey could be appropriate for this context but the depth and quality of the information gained here would not have been plausible with a survey design.

\section{CONCLUSIONS}

This study has highlighted a number of implications for community pharmacy practice. The authors suggest the development or adaptation of resources for use in community pharmacy including a resource to assist pharmacists and pharmacy staff in providing self-care advice to patients for common infections, a resource to assist pharmacists and pharmacy staff in giving antibiotic compliance advice to patients and audits for pharmacists and pharmacy staff to monitor and improve self-care advice and antibiotic compliance advice.

\footnotetext{
Author affiliations

${ }^{1}$ Primary Care Unit, Public Health England, Gloucester, UK

${ }^{2}$ Behavioural Insights Team, Public Health England, London, UK

${ }^{3} \mathrm{HCAl} \&$ AMR, Public Health England, London, UK

${ }^{4}$ Boots UK, Nottingham, Nottingham, UK
}

${ }^{5}$ School of Medicine, University Hospital of Wales, Cardiff, UK

${ }^{6}$ Nuffield Department of Primary Care Health Sciences, Oxford University, Oxford, UK

Acknowledgements The study team wish to thank all of the participants who agreed to take part in focus groups and interviews for the study. Thank you to Charlotte Eley of Public Health England's Primary Care Unit for conducting the second coding for this study. Thank you to Margaret Heginbothom and her team for helping with recruitment in Wales.

Contributors LFJ commented on the protocol, conducted all interviews and focus groups, conducted the data analysis, and wrote the paper. R0 wrote the protocol with CAMM, oversaw the day-to-day running of the study and commented on the paper. AS commented on the protocol, adapted the interview schedule to the TDF, assisted with data analysis and commented on the paper. DA- 0 commented on the protocol, assisted with recruitment and commented on the paper. TT worked with CAMM to devise the project, commented on the protocol, assisted with recruitment and commented on the paper. NAF commented on the protocol, assisted with recruitment and commented on the paper. $\mathrm{CB}$ commented on the protocol and commented on the paper. CAMM wrote the first draft of the protocol, devised and oversaw the entire study.

Funding The authors have not declared a specific grant for this research from any funding agency in the public, commercial or not-for-profit sectors.

Competing interests LFJ works for Public Health England's Primary Care Unit on the development of the TARGET Antibiotics resources for primary care clinicians. CAMM leads Public Health England's Primary Care Unit and leads the TARGET Antibiotic resources for primary care clinicians and Public Health England's quick reference antibiotic and diagnostic guides. TT leads pharmacy practice research for Boots UK and is honorary professor at the School of Pharmacy, University of Nottingham.

Patient consent for publication Not required.

Ethics approval Ethical approval was obtained from Cardiff University ethics committee (SMREC: 15/55).

Provenance and peer review Not commissioned; externally peer reviewed.

Data sharing statement All qualitative data from this study are held by Public Health England, Primary Care Unit.

Open access This is an open access article distributed in accordance with the Creative Commons Attribution Non Commercial (CC BY-NC 4.0) license, which permits others to distribute, remix, adapt, build upon this work non-commercially, and license their derivative works on different terms, provided the original work is properly cited, appropriate credit is given, any changes made indicated, and the use is non-commercial. See: http://creativecommons.org/licenses/by-nc/4.0/.

\section{REFERENCES}

1. World Health Organisation. Antimicrobial Resistance Global Report on Surveillance, 2014.

2. Davey P, Pagliari C, Hayes A. The patient's role in the spread and control of bacterial resistance to antibiotics. Clin Microbiol Infect 2002;8 Suppl 2:43-68.

3. Howard P, Ashiru-Oredope D, Gilchrist M. Time for pharmacy to unite in the fight against antimicrobial resistance. The Pharmaceutical Journal: The Royal Pharmaceutical Society 2013.

4. Jethwa S. Tackling antimicrobial resistance through education. Prescriber 2015;26:35-6.

5. Department of Health. Choosing health through pharmacy. 20052015.

6. Department of Health. Community pharmacy in 2016/17 and beyond. 2016.

7. Public Health England. Healthy Living Pharmacy Level 1 Quality Criteria, 2016.

8. Agomo CO. The role of community pharmacists in public health: a scoping review of the literature. Journal of Pharmaceutical Health Services Research 2012;3:25-33.

9. Northey A, McGuren T, Stupans I. Patients' antibiotic knowledge: a trial assessing the impact of verbal education. Int $J$ Pharm Pract 2015;23:158-60.

10. NHS England. Urgent and Emergency Care Review End of Phase 1 Report. In. Transforming urgent and emergency care services in England, 2013.

11. Choose Pharmacy. [http://www.wcppe.org.uk/learning/choosepharmacy] 
12. Written Statement - Investing in community pharmacy in Wales. http://gov.wales/about/cabinet/cabinetstatements/previousadministration/2016/communitypharmacy/?lang=en .

13. Postcode Area [http://www.postcodearea.co.uk/]

14. Cane J, O'Connor D, Michie S. Validation of the theoretical domains framework for use in behaviour change and implementation research. Implementation Science 2012;7:37.

15. Michie S, Atkins L, West R. The Behaviour Change Wheel, A Guide To Designing Interventions, 2014.

16. Pharmacy Mnemonics: WWHAM, ASMETHOD, ENCORE and SIT DOWN SIR. http://www.resourcepharm.com/pre-reg-pharmacist/ pharmacy-mnemonics.html.

17. Ashiru-Oredope D, Doble A, Thornley T. Klinger C: Antimicrobial stewardship and self-care advice in pharmacies (AMSAP) study; pilot implementation of TARGET community pharmacy RTI leaflet: In. International Pharmacy Congress, 2018.
18. Leaflets to share with patients: The TARGET Antibiotics Toolkit. http://www.rcgp.org.uk/clinical-and-research/toolkits/ /link.aspx? id=9FCF9DA4B4A045519593320478DFD9E7\&_z=z.

19. Caring for Children with Coughs. Information and Advice for Parents.

20. Quality improvement. http://careers.bmj.com/careers/advice/Quality improvement.

21. The Royal Pharmaceutical Society. Pharmacist access to the Patient Health Record, 2014.

22. Voice P. The non-supply of over-the-counter (OTC) products to people seeking self care, 2016.

23. Courtenay M, Rowbotham S, Lim R, et al. Antibiotics for acute respiratory tract infections: a mixed-methods study of patient experiences of non-medical prescriber management. BMJ Open 2017;7:e013515 